\title{
The Maximal Total Irregularity of Bicyclic Graphs
}

\author{
Lihua You, ${ }^{1}$ Jieshan Yang, ${ }^{1}$ Yingxue Zhu, ${ }^{1}$ and Zhifu You ${ }^{2}$ \\ ${ }^{1}$ School of Mathematical Sciences, South China Normal University, Guangzhou 510631, China \\ ${ }^{2}$ Department of Computer Science, Guangdong Polytechnic Normal University, Guangzhou 510665, China
}

Correspondence should be addressed to Lihua You; ylhua@scnu.edu.cn

Received 13 October 2013; Revised 27 February 2014; Accepted 11 March 2014; Published 10 April 2014

Academic Editor: Frank Werner

Copyright (C) 2014 Lihua You et al. This is an open access article distributed under the Creative Commons Attribution License, which permits unrestricted use, distribution, and reproduction in any medium, provided the original work is properly cited.

In 2012, Abdo and Dimitrov defined the total irregularity of a graph $G=(V, E)$ as $\operatorname{irr}_{t}(G)=(1 / 2) \sum_{u, v \in V}\left|d_{G}(u)-d_{G}(v)\right|$, where $d_{G}(u)$ denotes the vertex degree of a vertex $u \in V$. In this paper, we investigate the total irregularity of bicyclic graphs and characterize the graph with the maximal total irregularity among all bicyclic graphs on $n$ vertices.

\section{Introduction}

Let $G=(V, E)$ be a simple undirected graph with vertex set $V$ and edge set $E$. For any vertices $u, v \in V$, the degree of $v$ is denoted by $d_{G}(v)$, and the distance $d_{G}(u, v)$ is defined as the length of the shortest path between $u$ and $v$ in $G$. Let $P_{n}, C_{n}$, and $S_{n}$ be the path, cycle, and star on $n$ vertices, respectively.

A graph is regular if all its vertices have the same degree; otherwise it is irregular. Several approaches that characterize how irregular a graph is have been proposed. In [1], Albertson defined the imbalance of an edge $e=u v \in E$ as $\left|d_{G}(u)-d_{G}(v)\right|$ and the irregularity of $G$ as

$$
\operatorname{irr}(G)=\sum_{u v \in E}\left|d_{G}(u)-d_{G}(v)\right| .
$$

More results on the imbalance and the irregularity of a graph $G$ can be found in [1-4].

Inspired by the structure and meaning of (1), Abdo and Dimitrov [5] introduced a new irregularity measure, called the total irregularity. For a graph $G$, it is defined as

$$
\operatorname{irr}_{t}(G)=\frac{1}{2} \sum_{u, v \in V}\left|d_{G}(u)-d_{G}(v)\right| .
$$

Although the two irregularity measures capture the irregularity only by a single parameter, namely, the degree of a vertex, the new measure is more superior than the old one in some aspects. For example, (2) has an expected property of an irregularity measure that graphs with the same degree sequence have the same total irregularity, while (1) does not have. Both measures also have common properties, including that they are zero if and only if $G$ is regular.

Obviously, $\operatorname{irr}_{t}(G)$ is an upper bound of $\operatorname{irr}(G)$. In [6], the authors derived relation between $\operatorname{irr}_{t}(G)$ and $\operatorname{irr}(G)$ for a connected graph $G$ with $n$ vertices; that is, $\operatorname{irr}_{t}(G) \leq n^{2}$ $\operatorname{irr}(G) / 4$. Furthermore, they showed that $\operatorname{irr}_{t}(T) \leq(n-2)$ $\operatorname{irr}(T)$ for any tree $T$.

In [5], the authors obtained the upper bound of the total irregularity among all graphs with $n$ vertices, and they showed that the star graph $S_{n}$ is the tree with the maximal total irregularity among all trees with $n$ vertices.

Theorem 1 (see [5]). Let $G$ be a simple, undirected graph on $n$ vertices. Then,

(1) $\operatorname{irr}_{t}(G) \leq(1 / 12)\left(2 n^{3}-3 n^{2}-2 n+3\right)$.

(2) If $G$ is a tree, then $\operatorname{irr}_{t}(G) \leq(n-1)(n-2)$, with equality holds if and only if $G \cong S_{n}$.

In [7], the authors investigated the total irregularity of unicyclic graphs and determined the graph with the maximal total irregularity $n^{2}-n-6$ among unicyclic graphs on $n$ vertices. In [8], the authors investigated the minimal total irregularity of graphs, and they characterized the graph with the minimal, the second minimal, the third minimal total irregularity among trees, unicyclic graphs or bicyclic graphs on $n$ vertices. 


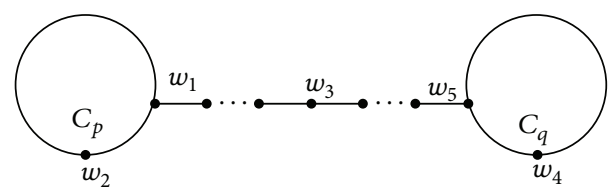

FIgURE 1: The graph $\infty(p, q, l)$ with $l \geq 3$.

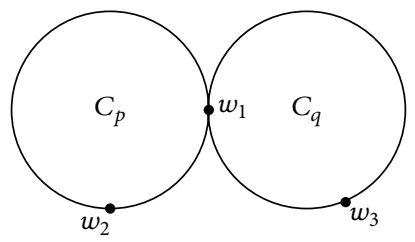

FIGURE 2: The graph $\infty(p, q, 1)$.

Recently, Abdo and Dimitrov [9] also obtained the upper bounds on the total irregularity of graphs under several graph operations including join, lexicographic product, Cartesian product, strong product, direct product, corona product, disjunction, and symmetric difference.

In this paper, we introduce two transformations to study the total irregularity of bicyclic graphs in Section 2 and characterize the graph with the maximal total irregularity $n^{2}+n-16$ among all bicyclic graphs on $n$ vertices in Section 3 .

\section{Some Preliminaries and Two Transformations}

In this section, we introduce some definitions, notations, two transformations, and basic properties which we need to use in the proofs of the main results. Other undefined notations may refer to [10].

A bicyclic graph is a simple connected graph in which the number of edges equals the number of vertices plus one. There are two basic bicyclic graphs: $\infty$-graph and $\Theta$-graph. An $\infty$-graph, denoted by $\infty(p, q, l)$ (see Figure 1 ), is obtained from two vertex-disjoint cycles $C_{p}$ and $C_{q}$ by connecting one vertex of $C_{p}$ and one of $C_{q}$ with a path $P_{l}$ of length $l-1$ (in the case of $l=1$, identifying the above two vertices, see Figure 2) where $p, q \geq 3$ and $l \geq 1$; and a $\Theta$-graph, denoted by $\theta(p, q, l)$ (see Figure 3), is a graph on $p+q-l$ vertices with the two cycles $C_{p}$ and $C_{q}$ having $l$ common vertices, where $p, q \geq 3$ and $l \geq 2$.

In Figure 1, let $w_{1}\left(w_{5}\right)$ be the common vertex of $P_{l}$ and $C_{p}\left(C_{q}\right) ; w_{2} \in V\left(C_{p}\right) \backslash\left\{w_{1}\right\}$ and $w_{3} \in V\left(P_{l}\right) \backslash\left\{w_{1}, w_{5}\right\}$ if $l \geq 3$, $w_{4} \in V\left(C_{q}\right) \backslash\left\{w_{5}\right\}$.

In Figure 2, let $w_{1}=V\left(C_{p}\right) \cap V\left(C_{q}\right), w_{2} \in V(\infty(p, q, 1)) \backslash$ $V\left(C_{q}\right)$, and $w_{3} \in V(\infty(p, q, 1)) \backslash V\left(C_{p}\right)$.

In Figure 3, let $w_{1}=z_{1}, w_{2} \in\left\{x_{1}, x_{2}, \ldots, x_{p-l}\right\}, w_{3} \in$ $\left\{z_{2}, \ldots, z_{l-1}\right\}$ if $l \geq 3, w_{4} \in\left\{y_{1}, y_{2}, \ldots, y_{q-l}\right\}$, and $w_{5}=z_{l}$.

A rooted graph has one of its vertices, called the root, distinguished from the others. Let $T_{i}$ be a rooted tree with $\left|V\left(T_{i}\right)\right| \geq 2$ and root $v_{i}(1 \leq i \leq k)$.

Let $\mathbb{P}^{*}=\{P \mid P$ is a rooted path with length at least one and the root is its starting vertex $\}, \mathbb{S}^{*}=\{S \mid S$ is a rooted star and the root is its center $\}$, and $\mathbb{P} \mathbb{S}^{*}=\left\{P+S \mid P \in \mathbb{P}^{*}\right.$
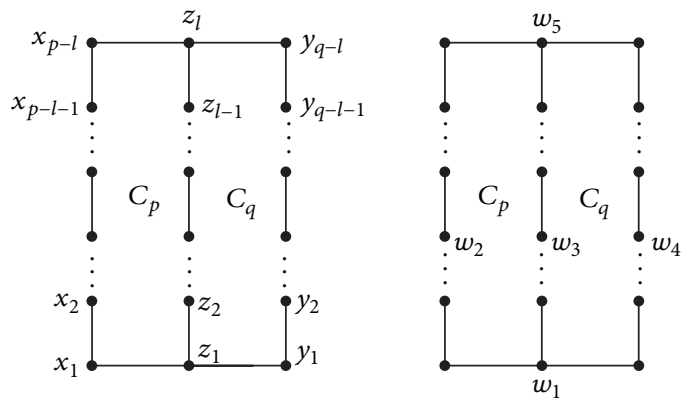

Figure 3: The graph $\theta(p, q, l)$.

and $S$ is a star\}, where the rooted graph $P+S$ is obtained by identifying the end vertex of $P \in \mathbb{P}^{*}$ with the center of a star $S$ and the root of $P+S$ is the root of $P$.

To identify nonadjacent vertices $x$ with $y$ of a graph $G$ is to replace these two vertices by a single vertex incident to all the edges which were incident in $G$ to either $x$ or $y$. Let $G_{1}$ and $G_{2}$ be two graphs: $v_{1} \in V\left(G_{1}\right)$ and $v_{2} \in V\left(G_{2}\right)$. The graph $G=\left(G_{1}, v_{1}\right) \Delta\left(G_{2}, v_{2}\right)$ denotes the graph resulting from identifying $v_{1}$ with $v_{2}$. Let $x \in V(\infty(p, q, l)), y \in V(\theta(p, q, l))$, and $v$ be the root of the rooted tree $T$. Take $\infty(p, q, l, T)=$ $(\infty(p, q, l), x) \Delta(T, v)$ and $\theta(p, q, l, T)=(\theta(p, q, l), y) \Delta(T, v)$. For example, see Figure 4.

\section{1. $\alpha$-Transformation}

$\alpha$-Transformation. Let $G=(V, E)$ be a bicyclic graph obtained from $\infty(p, q, l)(\theta(p, q, l))$ with $k(\geq 1)$ rooted trees $T_{1}, \ldots, T_{k}$ attached, and let $u \in V$ be one of the maximal degree vertices of $G$ and let $w$ be any pendent vertex of $G$ which is adjacent to vertex $y(y \neq u)$. Let $G^{\prime}$ be the graph obtained from $G$ by deleting the pendent edge $y w$ and adding a pendent edge $u w$. We call the transformation from $G$ to $G^{\prime}$ an $\alpha$-transformation on $G$. For example, see Figure 5.

Remark 2. Note that in Figure 5, the edge $y w \in E\left(T_{i}\right)$. In fact, $y w \in E\left(T_{j}\right)$ for any $j \in\{1, \ldots, k\}$.

Lemma 3. Let $G=(V, E)$ be a bicyclic graph obtained from $\infty(p, q, l)(\theta(p, q, l))$ with $k(\geq 1)$ rooted trees $T_{1}, \ldots, T_{k}$ attached, and let $G^{\prime}$ be the graph obtained from $G$ by $\alpha$ transformation as above. Then, $\operatorname{irr}_{t}(G)<\operatorname{irr}_{t}\left(G^{\prime}\right)$.

Proof. Note that after the $\alpha$-transformation on $G$, only the degrees of $u$ and $y$ have been changed; namely, $d_{G^{\prime}}(u)=$ $d_{G}(u)+1, d_{G^{\prime}}(y)=d_{G}(y)-1$, and $d_{G^{\prime}}(x)=d_{G}(x)$ for any $x \in V \backslash\{u, y\}$. Clearly,

$$
\begin{gathered}
\left|d_{G^{\prime}}(u)-d_{G^{\prime}}(y)\right|-\left|d_{G}(u)-d_{G}(y)\right|=2, \\
\sum_{x \in V \backslash\{u, y\}}\left(\left|d_{G^{\prime}}(u)-d_{G^{\prime}}(x)\right|-\left|d_{G}(u)-d_{G}(x)\right|\right)=n-2, \\
\sum_{x \in \backslash \backslash\{u, y\}}\left(\left|d_{G^{\prime}}(y)-d_{G^{\prime}}(x)\right|-\left|d_{G}(y)-d_{G}(x)\right|\right) \geq-(n-2)
\end{gathered}
$$




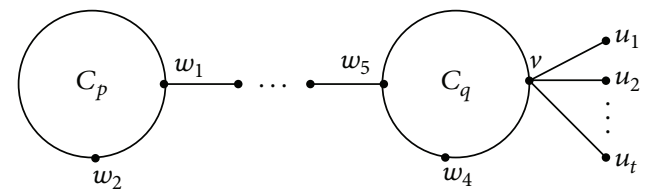

(a) The graph $\infty(p, q, l, T)$ where $T \in \mathbb{S}^{*}$ and $l \geq 2$

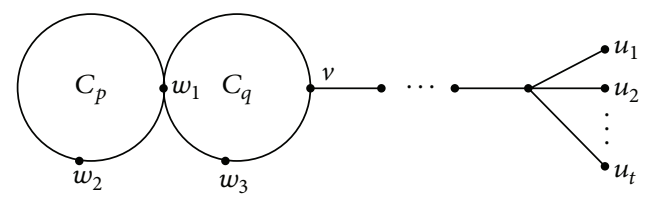

(b) The graph $\infty(p, q, 1, T)$ where $T \in \mathbb{P S}^{*}$

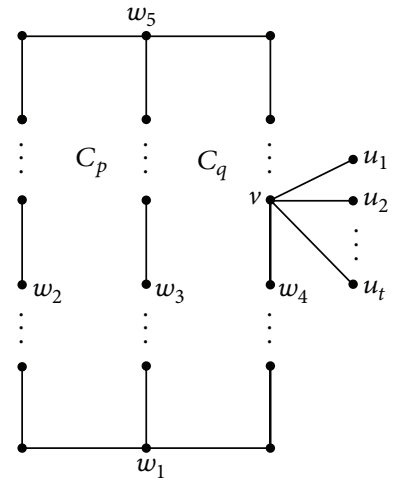

(c) The graph $\theta(p, q, l, T)$ where $T \in \mathbb{S}^{*}$

FIGURE 4: Three graphs.

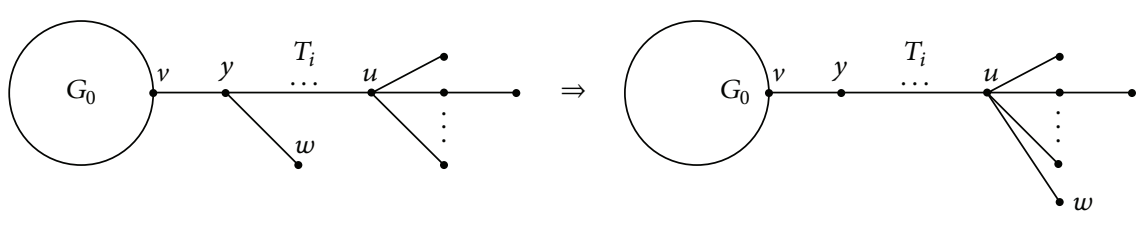

$G(p, q, k)$

$G^{\prime}$

FIGURE 5: $\alpha$-transformation.

because for any integer $a,|a-1|-|a|=\left\{\begin{array}{cc}-1, & \text { if } a>0 ; \\ 1, & \text { if } a \leq 0\end{array}\right.$. Then,

$$
\begin{aligned}
& \operatorname{irr}_{t}\left(G^{\prime}\right)-\operatorname{irr}_{t}(G) \\
& =\left(\left|d_{G^{\prime}}(u)-d_{G^{\prime}}(y)\right|-\left|d_{G}(u)-d_{G}(y)\right|\right) \\
& \quad+\sum_{x \in V \backslash\{u, y\}}\left(\left|d_{G^{\prime}}(u)-d_{G^{\prime}}(x)\right|-\left|d_{G}(u)-d_{G}(x)\right|\right) \\
& \quad+\sum_{x \in V \backslash\{u, y\}}\left(\left|d_{G^{\prime}}(y)-d_{G^{\prime}}(x)\right|-\left|d_{G}(y)-d_{G}(x)\right|\right) \\
& \geq 2+(n-2)+(-n+2)>0 .
\end{aligned}
$$

By Lemma 3 and the definition of $\alpha$-transformation, we have the following result.

Lemma 4. Let $G=(V, E)$ be a bicyclic graph obtained from $\infty(p, q, l)(\theta(p, q, l))$ with $k(\geq 1)$ rooted trees $T_{1}, \ldots, T_{k}$ attached.

(1) If one cannot get a new graph from $G$ by $\alpha$ transformation, then there exists some rooted tree $T$ such that $G \cong \infty(p, q, l, T)(G \cong \theta(p, q, l, T))$, where $T \in \mathbb{S}^{*} \cup \mathbb{P} \mathbb{S}^{*}$.

(2) Let $G_{1}$ be the graph obtained from $G$ by repeating $\alpha$ transformation, and one cannot get other graphs from $G_{1}$ by repeating $\alpha$-transformation. Then there exists some rooted tree $T$ such that $G_{1} \cong \infty(p, q, l, T)\left(G_{1} \cong\right.$ $\theta(p, q, l, T))$, where $T \in \mathbb{S}^{*} \cup \mathbb{P S}^{*}$ and $\operatorname{irr}_{t}(G)<$ $\operatorname{irr}_{t}\left(G_{1}\right)$.

\section{2. $\beta$-Transformation}

$\beta$-Transformation. Let $G=(V, E)$ be a bicyclic graph and $G \cong \infty(p, q, l, T)(G \cong \theta(p, q, l, T))$, where $T \in \mathbb{S}^{*} \cup \mathbb{P} \mathbb{S}^{*}$, let $v$ be the root of the unique rooted tree $T$, let $u$ be a vertex of $T$ and let $u_{1}, u_{2}, \ldots, u_{t}(t \geq 1)$ be the pendent vertices adjacent to $u$. Let $G^{\prime}$ be the graph obtained from $G$ by deleting the pendent edges $u u_{1}, u u_{2}, \ldots, u u_{t}$ and adding pendent edges $w_{1} u_{1}, w_{1} u_{2}, \ldots, w_{1} u_{t}$. We call the transformation from $G$ to $G^{\prime}$ a $\beta$-transformation on $G$. For example, see Figures 6-8.

Lemma 5. Let $G=\infty(p, q, l, T)(G=\theta(p, q, l, T))$ be a bicyclic graph where $T \in \mathbb{P} \mathbb{S}^{*}$, let $v$ be the root of the unique rooted tree $T$, let $u$ be a vertex of $T$ and let $u_{1}, u_{2}, \ldots, u_{t}(t \geq 1)$ be the pendent vertices adjacent to $u$. Let $G^{\prime}$ be the graph obtained from $G$ by $\beta$-transformation (e.g., see Figure 7). Then, $\operatorname{irr}_{t}(G)<\operatorname{irr}_{t}\left(G^{\prime}\right)$.

Proof. For convenience, let $G=(V, E)$. Note that the root of the unique rooted tree, $v$, is not necessarily different with $w_{1}$. Clearly, we know that only the degrees of $u$ and $w_{1}$ have been changed after the $\beta$-transformation; namely, $d_{G^{\prime}}(u)=1$, $d_{G^{\prime}}\left(w_{1}\right)=d_{G}\left(w_{1}\right)+d_{G}(u)-1$, and $d_{G^{\prime}}(x)=d_{G}(x)$ for any vertex $x \in V \backslash\left\{u, w_{1}\right\}$. Let $U=V \backslash\left\{u, w_{1}\right\}$. Note that $l+t \geq 2$; then we complete the proof by the following three cases.

Case $1(2 \leq l+t \leq 3)$. In this case, $d_{G}(u)<d_{G}\left(w_{1}\right)$. It implies that the $\beta$-transformation on $G$ is $\alpha$-transformation $t$ times on $G$. Then, $\operatorname{irr}_{t}(G)<\operatorname{irr}_{t}\left(G^{\prime}\right)$ by using Lemma $3 t$ times.

Case $2(l+t \geq 4, t=1)$. The proof is similar to the proof of Case 1; thus, we omit it. 


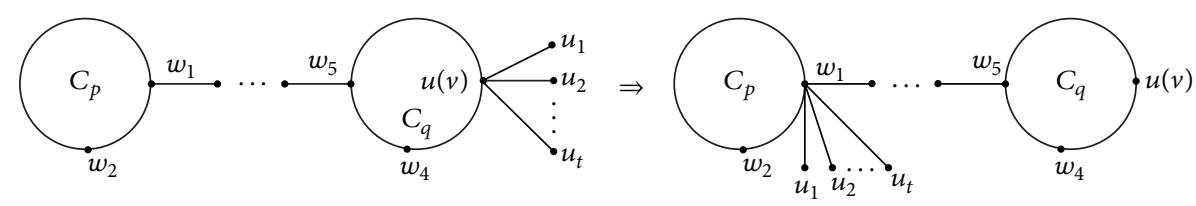

Figure 6: $\beta$-transformation on $\infty(p, q, l, T)$ with $T \in \mathbb{S}^{*}$.

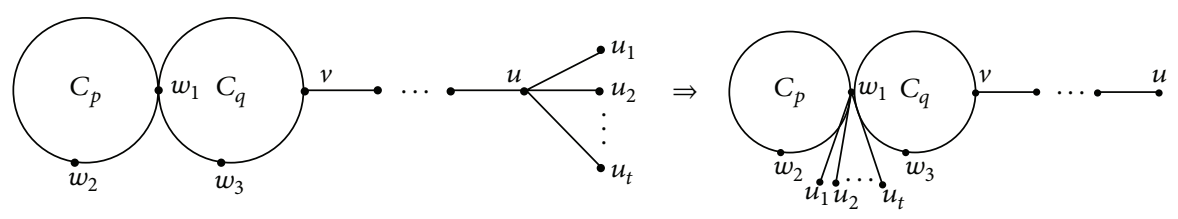

FIGURE 7: $\beta$-Transformation on $\infty(p, q, l, T)$ with $T \in \mathbb{P S}^{*}$.

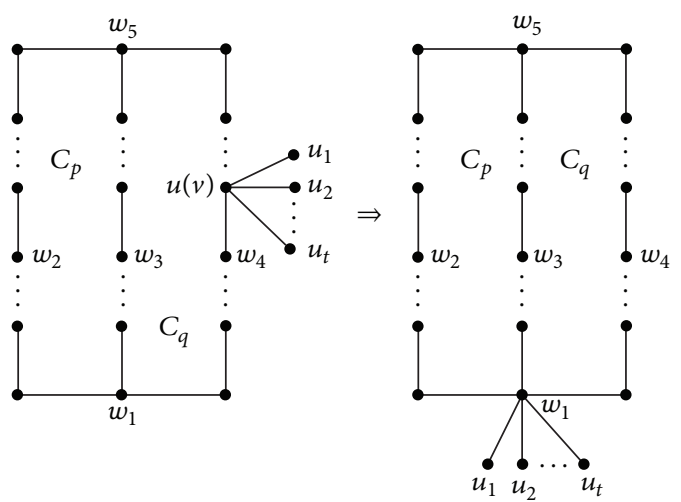

Figure 8: $\beta$-Transformation on $\theta(p, q, l, T)$ where $T \in \mathbb{S}^{*}$.

Case $3(l+t \geq 4, t \geq 2)$. In this case, vertex $u$ is one of the maximal degree vertices of $G$; namely, $d_{G}(u) \geq d_{G}(x)$ for any vertex $x \in V \backslash\{u\}$. Then,

$$
\begin{gathered}
\left|d_{G^{\prime}}(u)-d_{G^{\prime}}\left(w_{1}\right)\right|-\left|d_{G}(u)-d_{G}\left(w_{1}\right)\right|=2 d\left(w_{1}\right)-2, \\
\sum_{x \in U}\left|d_{G^{\prime}}(u)-d_{G^{\prime}}(x)\right|-\sum_{x \in U}\left|d_{G}(u)-d_{G}(x)\right| \\
=2 \sum_{x \in U} d_{G}(x)-(n-2)\left(d_{G}(u)+1\right) .
\end{gathered}
$$

Now we discuss $\sum_{x \in U}\left|d_{G^{\prime}}\left(w_{1}\right)-d_{G^{\prime}}(x)\right|-\sum_{x \in U} \mid d_{G}\left(w_{1}\right)-$ $d_{G}(x) \mid$ as follows.

Subcase $3.1(t \geq 2, l \geq 2)$. Because $d_{G}\left(w_{1}\right)-d_{G}\left(w_{5}\right)-\mid d_{G}\left(w_{1}\right)-$

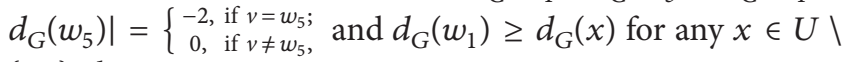
$\left\{w_{5}\right\}$, then

$$
\begin{gathered}
\sum_{x \in U}\left|d_{G^{\prime}}\left(w_{1}\right)-d_{G^{\prime}}(x)\right|-\sum_{x \in U}\left|d_{G}\left(w_{1}\right)-d_{G}(x)\right| \\
=\sum_{x \in U \backslash\left\{w_{5}\right\}}\left(d_{G}\left(w_{1}\right)+d_{G}(u)-1-d_{G}(x)\right)
\end{gathered}
$$

$$
\begin{aligned}
& -\sum_{x \in U \backslash\left\{w_{5}\right\}}\left(d_{G}\left(w_{1}\right)-d_{G}(x)\right) \\
& +\left(d_{G}\left(w_{1}\right)+d_{G}(u)-1-d_{G}\left(w_{5}\right)\right) \\
& -\left|d_{G}\left(w_{1}\right)-d_{G}\left(w_{5}\right)\right| \\
= & (n-2)\left(d_{G}(u)-1\right)+\left(d_{G}\left(w_{1}\right)-d_{G}\left(w_{5}\right)\right) \\
& -\left|d_{G}\left(w_{1}\right)-d_{G}\left(w_{5}\right)\right| \\
\geq & (n-2)\left(d_{G}(u)-1\right)-2 .
\end{aligned}
$$

Subcase $3.2(t \geq 3, l=1)$. Because $d_{G}\left(w_{1}\right) \geq d_{G}(x)$ for any $x \in U$, then

$$
\begin{aligned}
\sum_{x \in U} \mid & d_{G^{\prime}}\left(w_{1}\right)-d_{G^{\prime}}(x)\left|-\sum_{x \in U}\right| d_{G}\left(w_{1}\right)-d_{G}(x) \mid \\
= & \sum_{x \in U}\left(d_{G}\left(w_{1}\right)+d_{G}(u)-1-d_{G}(x)\right) \\
& -\sum_{x \in U}\left(d_{G}\left(w_{1}\right)-d_{G}(x)\right)=(n-2)\left(d_{G}(u)-1\right) .
\end{aligned}
$$

Combining the above two subcases, we have

$$
\begin{aligned}
& \sum_{x \in U}\left|d_{G^{\prime}}\left(w_{1}\right)-d_{G^{\prime}}(x)\right|-\sum_{x \in U}\left|d_{G}\left(w_{1}\right)-d_{G}(x)\right| \\
& \quad \geq(n-2)\left(d_{G}(u)-1\right)-2 .
\end{aligned}
$$

By $d_{G}\left(w_{1}\right) \geq 3$ and $d_{G}(x) \geq 1$ for any $x \in U$ and the above arguments, we have

$$
\begin{aligned}
\operatorname{irr}_{t}\left(G^{\prime}\right)-\operatorname{irr}_{t}(G) \\
=\left|d_{G^{\prime}}(u)-d_{G^{\prime}}\left(w_{1}\right)\right| \\
\quad+\sum_{x \in U}\left|d_{G^{\prime}}(u)-d_{G^{\prime}}(x)\right|+\sum_{x \in U}\left|d_{G^{\prime}}\left(w_{1}\right)-d_{G^{\prime}}(x)\right|
\end{aligned}
$$




$$
\begin{aligned}
& -\left[\left|d_{G}(u)-d_{G}\left(w_{1}\right)\right|+\sum_{x \in U}\left|d_{G}(u)-d_{G}(x)\right|\right. \\
& \left.\quad+\sum_{x \in U}\left|d_{G}\left(w_{1}\right)-d_{G}(x)\right|\right] \\
& \geq 2 d_{G}\left(w_{1}\right)-2+2 \sum_{x \in U} d_{G}(x) \\
& -(n-2)\left(d_{G}(u)+1\right)+(n-2)\left(d_{G}(u)-1\right)-2 \geq 2 .
\end{aligned}
$$

Let $G=\infty(p, q, l, T)(G=\theta(p, q, l, T))$ be a bicyclic graph, let $w_{i} \in V(G)=V(i=1, \ldots, 5)$ as defined in Figures $1-3$, and let $u$ be the root of $T$. Take

$$
\begin{array}{r}
\infty^{i}(p, q, l, T)=\left(\infty(p, q, l), w_{i}\right) \Delta(T, u), \\
\text { where } l \geq 2, i \in\{1,2,3,4,5\}, \\
\infty^{j}(p, q, 1, T)=\left(\infty(p, q, 1), w_{j}\right) \Delta(T, u), \\
\text { where } j \in\{1,2,3\}, \\
\theta^{r}(p, q, l, T)=\left(\theta(p, q, l), w_{r}\right) \Delta(T, u), \\
\text { where } r \in\{1,2,3,4,5\} .
\end{array}
$$

Clearly, $\theta^{1}(p, q, l, T) \cong \theta^{5}(p, q, l, T)$.

Lemma 6. Let $G=\infty(p, q, l, T)(G=\theta(p, q, l, T))$ be a bicyclic graph, where $T \in \mathbb{S}^{*}$ and $G \neq \infty^{1}(p, q$, $l, T)\left(G \neq \theta^{1}(p, q, l, T)\right)$, let $u$ be the root of the unique rooted tree $T$, and let $u_{1}, u_{2}, \ldots, u_{t}(t \geq 1)$ be the pendent vertices adjacent to $u$. Let $G^{\prime}$ be the graph obtained from $G$ by $\beta$-transformation (e.g., see Figures 6 and 8). Then,

(1) $G^{\prime}=\infty^{1}(p, q, l, T)\left(G^{\prime}=\theta^{1}(p, q, l, T)\right)$.

(2) $\operatorname{irr}_{t}(G) \leq \operatorname{irr}_{t}\left(G^{\prime}\right)$, and the equality holds if and only if $G=\infty^{5}(p, q, l, T)\left(G=\theta^{5}(p, q, l, T)\right)$.

Proof. Clearly, $u \neq w_{1}, \quad$ since $\quad G \neq \infty^{1}(p, q, l, T)\left(G \neq \theta^{1}\right.$ $(p, q, l, T))$.

By the definition of $\beta$-transformation, (1) is obvious. Now we show that (2) holds.

If $u=w_{5}$ when $l \geq 2$, namely, $G=\infty^{5}(p, q, l, T)(G=$ $\left.\theta^{5}(p, q, l, T)\right)$, then $G$ and $G^{\prime}$ have the same degree sequence; thus they have the same total irregularity: $\operatorname{irr}_{t}(G)=\operatorname{irr}_{t}\left(G^{\prime}\right)$.

Hence, we assume $u \notin\left\{w_{1}, w_{5}\right\}$ in the following. Note that only the degrees of vertices $u$ and $w_{1}$ have been changed after the $\beta$-transformation; namely, $d_{G^{\prime}}(u)=2, d_{G^{\prime}}\left(w_{1}\right)=$ $d_{G}\left(w_{1}\right)+d_{G}(u)-2$, and $d_{G^{\prime}}(x)=d_{G}(x)$ for any vertex $x \in$ $V \backslash\left\{u, w_{1}\right\}$. Let $U=V \backslash\left\{u, w_{1}\right\}$. Note that $l+t \geq 2$; then we complete the proof by the following two cases.
Case $1(l+t \geq 3)$. In this case, vertex $u$ is one of the maximal degree vertices of $G$; namely, $d_{G}(u) \geq d_{G}(x)$ for any vertex $x \in V \backslash\{u\}$ and $d_{G}\left(w_{1}\right) \geq d_{G}(x)$ for any $x \in U$. Then,

$$
\begin{gathered}
\left|d_{G^{\prime}}(u)-d_{G^{\prime}}\left(w_{1}\right)\right|-\left|d_{G}(u)-d_{G}\left(w_{1}\right)\right|=2 d_{G}\left(w_{1}\right)-4, \\
\sum_{x \in U}\left|d_{G^{\prime}}\left(w_{1}\right)-d_{G^{\prime}}(x)\right|-\sum_{x \in U}\left|d_{G}\left(w_{1}\right)-d_{G}(x)\right| \\
=(n-2)\left(d_{G}(u)-2\right), \\
\sum_{x \in U}\left|d_{G^{\prime}}(u)-d_{G^{\prime}}(x)\right|-\sum_{x \in U}\left|d_{G}(u)-d_{G}(x)\right| \\
=\sum_{x \in U}\left[\left|d_{G}(x)-2\right|-\left(d_{G}(u)-d_{G}(x)\right)\right] \\
\geq t+\sum_{x \in U} d_{G}(x)-(n-2) d_{G}(u) .
\end{gathered}
$$

By $d_{G}\left(w_{1}\right) \geq 3$ and $t=d_{G}(u)-2 \geq 1$ and the above arguments, we have

$$
\begin{aligned}
& \operatorname{irr}_{t}\left(G^{\prime}\right)-\operatorname{irr}_{t}(G) \\
& =\left|d_{G^{\prime}}(u)-d_{G^{\prime}}\left(w_{1}\right)\right|+\sum_{x \in U}\left|d_{G^{\prime}}(u)-d_{G^{\prime}}(x)\right| \\
& \quad+\sum_{x \in U}\left|d_{G^{\prime}}\left(w_{1}\right)-d_{G^{\prime}}(x)\right| \\
& \quad-\left[\left|d_{G}(u)-d_{G}\left(w_{1}\right)\right|+\sum_{x \in U}\left|d_{G}(u)-d_{G}(x)\right|\right. \\
& \left.\quad+\sum_{x \in U}\left|d_{G}\left(w_{1}\right)-d_{G}(x)\right|\right] \\
& \geq\left(2 d_{G}\left(w_{1}\right)-4\right)+(n-2)\left(d_{G}(u)-2\right)+t \\
& \quad+\sum_{x \in U} d_{G}(x)-(n-2) d_{G}(u) \geq 2 .
\end{aligned}
$$

Case $2(l+t=2)$. In this case, $d_{G}(u)<d_{G}\left(w_{1}\right)$. It implies that the $\beta$-transformation on $G$ is $\alpha$-transformation 1 time on $G$. Then, $\operatorname{irr}_{t}(G)<\operatorname{irr}_{t}\left(G^{\prime}\right)$ by Lemma 3 .

By the proof of Lemma 6, we obtain the following result.

Corollary 7. Let $T \in \mathbb{S}^{*}$. Then,

(1) $\operatorname{irr}_{t}\left(\infty^{1}(p, q, l, T)\right)=\operatorname{irr}_{t}\left(\infty^{5}(p, q, l, T)\right)=\max \left\{\operatorname{irr}_{t}\right.$ $\left.\left(\infty^{1}(p, q, l, T)\right), \ldots, \operatorname{irr}_{t}\left(\infty^{5}(p, q, l, T)\right)\right\}$, where $l \geq 2$.

(2) $\operatorname{irr}_{t}\left(\infty^{1}(p, q, 1, T)\right)=\max \left\{\operatorname{irr}_{t}\left(\infty^{1}(p, q, 1, T)\right), \operatorname{irr}_{t}\right.$ $\left.\left(\infty^{2}(p, q, 1, T)\right), \operatorname{irr}_{t}\left(\infty^{3}(p, q, 1, T)\right)\right\}$.

(3) $\operatorname{irr}_{t}\left(\theta^{1}(p, q, l, T)\right)=\operatorname{irr}_{t}\left(\theta^{5}(p, q, l, T)\right)=\max \left\{\operatorname{irr}_{t}\left(\theta^{1}\right.\right.$ $\left.(p, q, l, T)), \ldots, \operatorname{irr}_{t}\left(\theta^{5}(p, q, l, T)\right)\right\}$. 


\section{The maximal Total Irregularity of Bicyclic Graphs}

In this section, we will obtain the upper bound of the total irregularity among all bicyclic graphs on $n$ vertices and characterize the extremal graph.

Observe that any bicyclic graph $G$ is obtained from an $\infty$ graph or a $\Theta$-graph (possibly) by attaching trees to some of its vertices. Denoted by $\mathscr{B}_{n}$ is the set of all bicyclic graphs on $n$ vertices. Obviously, $\mathscr{B}_{n}$ consists of three types of graphs: first type, denoted by $B_{n}^{+}$, is the set of those graphs each of which is an $\infty$-graph, $\infty(p, q, l)$, with trees attached when $l=1$; second type, denoted by $B_{n}^{++}$, is the set of those graphs each of which is an $\infty$-graph, $\infty(p, q, l)$, with trees attached when $l \geq 2$; third type, denoted by $\Theta_{n}$, is the set of those graphs each of which is a $\Theta$-graph, $\theta(p, q, l)$, with trees attached. Then, $\mathscr{B}_{n}=B_{n}^{+} \cup B_{n}^{++} \cup \Theta_{n}$.

3.1. The Graph with the Maximal Total Irregularity in $B_{n}^{+}$. In this subsection, the bicyclic graph with the maximal total irregularity in $B_{n}^{+}$is determined.

Let $n, p, q, r$ be positive integers with $p, q \geq 3$ and $p+q+$ $r-2=n ; G=\infty^{1}\left(p, q, 1, S_{r}\right)$. Clearly, the degree sequence of $G$ is $(r+3,2, \ldots, 2,1, \ldots, 1)$. By simple calculation, we have

$$
\operatorname{irr}_{t}(G)=(n-p-q+1)(n+p+q)+2 p+2 q-4 .
$$

Theorem 8. Let $n, p, q, r$ be positive integers with $p, q \geq 3$ and $p+q+r-2=n$ and let $G \in B_{n}^{+}$be a bicyclic graph on $n$ vertices obtained from $\infty(p, q, 1)$ with $k(\geq 1)$ rooted trees $T_{1}, \ldots, T_{k}$ attached. Then, $\operatorname{irr}_{t}(G) \leq(n-p-q+1)(n+p+q)+2 p+2 q-4$, and the equality holds if and only if $G \cong \infty^{1}\left(p, q, 1, S_{r}\right)$.

Proof. If $r=1$, then $n=p+q-1$, and the rooted trees $T_{1}, \ldots, T_{k}$ are trivial; namely, $\left|V\left(T_{i}\right)\right|=1$ with $i=1, \ldots, k$. Then, $G=\infty(p, q, 1)=\infty^{1}\left(p, q, 1, S_{1}\right)$ and $\operatorname{irr}_{t}(G)=(n-p-$ $q+1)(n+p+q)+2 p+2 q-4$. cases.

If $r \geq 2$, we complete the proof by the following three

Case $1\left(G \neq \infty(p, q, 1, T)\right.$ where $\left.T \in \mathbb{S}^{*} \cup \mathbb{P} \mathbb{S}^{*}\right)$. Let $G_{1}$ be the graph obtained from $G$ by repeating $\alpha$-transformation, and we cannot get a new graph from $G_{1}$ by $\alpha$-transformation. Then there exists a rooted tree $T$ such that $G_{1} \cong \infty(p, q, 1, T)$ where $T \in \mathbb{S}^{*} \cup \mathbb{P} \mathbb{S}^{*}$ and $\operatorname{irr}_{t}(G)<\operatorname{irr}_{t}\left(G_{1}\right)$ by Lemma 4 . Let $u$ be the root of the rooted tree $T$.

Subcase $1.1\left(G_{1} \cong \infty(p, q, 1, T)\right.$ where $\left.T \in \mathbb{S}^{*}\right)$. If $u=w_{1}$, then $G_{1} \cong \infty^{1}\left(p, q, 1, S_{r}\right)$ and $\operatorname{irr}_{t}(G)<\operatorname{irr}_{t}\left(G_{1}\right)=(n-p-$ $q+1)(n+p+q)+2 p+2 q-4$.

If $u \neq w_{1}$, then we can get a new graph $G_{2} \cong \infty^{1}\left(p, q, 1, S_{r}\right)$ by $\beta$-transformation on $G_{1}$; thus $\operatorname{irr}_{t}(G)<\operatorname{irr}_{t}\left(G_{1}\right)<$ $\operatorname{irr}_{t}\left(G_{2}\right)=(n-p-q+1)(n+p+q)+2 p+2 q-4$ by Lemma 6 .

Subcase $1.2\left(G_{1} \cong \infty(p, q, 1, T)\right.$ where $\left.T \in \mathbb{P S}^{*}\right)$. If $u \neq w_{1}$, let $G_{2}$ be the graph obtained from $G_{1}$ by $\beta$-transformation. Then $\operatorname{irr}_{t}\left(G_{1}\right)<\operatorname{irr}_{t}\left(G_{2}\right)$ by Lemma 5. Repeating $\alpha$-transformation on $G_{2}$ until we cannot get a new graph, the resulting graph is $G_{3} \cong \infty^{1}\left(p, q, 1, S_{r}\right)$. By Lemma $4, \operatorname{irr}_{t}\left(G_{2}\right)<\operatorname{irr}_{t}\left(G_{3}\right)$ and

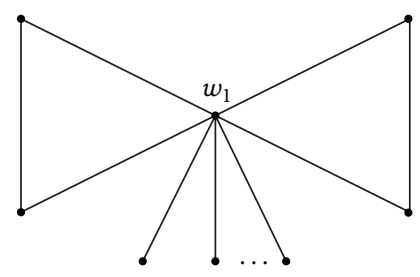

FIGURE 9: The graph $\infty^{1}\left(3,3,1, S_{n-4}\right)$.

thus $\operatorname{irr}_{t}(G)<\operatorname{irr}_{t}\left(G_{1}\right)<\operatorname{irr}_{t}\left(G_{2}\right)<\operatorname{irr}_{t}\left(G_{3}\right)=(n-p-q+$ 1) $(n+p+q)+2 p+2 q-4$.

If $u=w_{1}$ and $d_{G_{1}}(u, v)=1$, let $G_{2}$ be the graph obtained from $G_{1}$ by $\beta$-transformation; then $G_{2} \cong \infty^{1}\left(p, q, 1, S_{r}\right)$ and $\operatorname{irr}_{t}(G)<\operatorname{irr}_{t}\left(G_{1}\right)<\operatorname{irr}_{t}\left(G_{2}\right)=(n-p-q+1)(n+p+q)+$ $2 p+2 q-4$ by Lemma 5 .

If $u=w_{1}$ and $d_{G_{1}}(u, v)>1$, Let $G_{2}$ be the graph obtained from $G_{1}$ by $\beta$-transformation and let $G_{3}$ be the graph obtained from $G_{2}$ by repeating $\alpha$-transformation until we cannot get a new graph from $G_{3}$ by $\alpha$-transformation. Then by Lemmas 4 and 5 , we know that $G_{3} \cong \infty^{1}\left(p, q, 1, S_{r}\right)$ and $\operatorname{irr}_{t}(G)<$ $\operatorname{irr}_{t}\left(G_{1}\right)<\operatorname{irr}_{t}\left(G_{2}\right)<\operatorname{irr}_{t}\left(G_{3}\right)=(n-p-q+1)(n+p+$ q) $+2 p+2 q-4$

Case $2\left(G \cong \infty(p, q, 1, T)\right.$ where $\left.T \in \mathbb{S}^{*}\right)$.

The proof is similar to the proof of Subcase 1.1; thus, we omit it.

Case $3\left(G \cong \infty(p, q, 1, T)\right.$ where $\left.T \in \mathbb{P} \mathbb{S}^{*}\right)$.

The proof is similar to the proof of Subcase 1.2; thus, we omit it.

Combining the above arguments, we complete the proof.

Theorem 9. Let $p, q, r$ be positive integers with $p, q \geq 3$.

(1) If $p \geq 4$, then $\operatorname{irr}_{t}\left(\infty^{1}\left(p, q, 1, S_{r}\right)\right)<\operatorname{irr}_{t}\left(\infty^{1}(p-\right.$ $\left.\left.1, q, 1, S_{r+1}\right)\right)$.

(2) If $q \geq 4$, then $\operatorname{irr}_{t}\left(\infty^{1}\left(p, q, 1, S_{r}\right)\right)<\operatorname{irr}_{t}\left(\infty^{1}(p, q-\right.$ $\left.\left.1,1, S_{r+1}\right)\right)$.

Proof. Clearly, the proofs of (1) and (2) are similar; we only show that (1) holds. Let $n=p+q+r-2$; by formula (13), we have $\operatorname{irr}_{t}\left(\infty^{1}\left(p-1, q, 1, S_{r+1}\right)\right)-\operatorname{irr}_{t}\left(\infty^{1}\left(p, q, 1, S_{r}\right)\right)=2 p+$ $2 q-4>0$.

Theorem 10. Let $n \geq 5$ be a positive integer, and let $G \in B_{n}^{+}$ be a bicyclic graph on $n$ vertices. Then $\operatorname{irr}_{t}(G) \leq n^{2}+n-22$ and the equality holds if and only if $G \cong \infty^{1}\left(3,3,1, S_{n-4}\right)$ (see Figure 9).

Proof. Let $n, p, q, r$ be positive integers with $p, q \geq 3$ and $p+$ $q+r-2=n$ and let $G \in B_{n}^{+}$be a bicyclic graph on $n$ vertices obtained from $\infty(p, q, 1)$ with $k(\geq 1)$ rooted trees $T_{1}, \ldots, T_{k}$ attached. Then by Theorem 8 , we have $\operatorname{irr}_{t}(G) \leq(n-p-q+$ $1)(n+p+q)+2 p+2 q-4$, and the equality holds if and 
only if $G \cong \infty^{1}\left(p, q, 1, S_{r}\right)$. Now we complete the proof by the following three cases.

Case $1(p=q=3)$. Then, $\operatorname{irr}_{t}\left(\infty^{1}\left(3,3,1, S_{n-4}\right)\right)=n^{2}+n-22$.

Case $2(p \geq q \geq 3$ and $p \geq 4)$. Then by using Theorem $9 p+q-6$ times, we have $\operatorname{irr}_{t}\left(\infty^{1}\left(p, q, 1, S_{r}\right)\right)<$ $\operatorname{irr}_{t}\left(\infty^{1}\left(3, q, 1, S_{n-q-1}\right)\right) \leq \operatorname{irr}_{t}\left(\infty^{1}\left(3,3,1, S_{n-4}\right)\right)$.

Case 3 ( $q \geq p \geq 3$ and $q \geq 4$ ). The proof is similar to Case 2; thus, we omit it.

3.2. The Graph with the Maximal Total Irregularity in $B_{n}^{++}$. In this subsection, the bicyclic graph with the maximal total irregularity in $B_{n}^{++}$is determined.

Let $n, p, q, r, l$ be positive integers with $p, q \geq 3, l \geq 2$, and $p+q+r+l-3=n ; G=\infty^{1}\left(p, q, 1, S_{\mathrm{r}}\right)$ or $G=\infty^{5}\left(p, q, l, S_{r}\right)$. Clearly, the degree sequence of $G$ is $(r+2,3,2, \ldots, 2,1, \ldots, 1)$. By simple calculation, we have

$$
\begin{aligned}
\operatorname{irr}_{t}(G)= & (n-p-q-l+2)(n+p+q+l-1) \\
& +2 p+2 q+2 l-8 .
\end{aligned}
$$

Theorem 11. Let $n, p, q, l, r$ be positive integers with $p, q \geq 3$, $l \geq 2$, and $p+q+r+l-3=n$ and let $G \in B_{n}^{++}$be a bicyclic graph on $n$ vertices obtained from $\infty(p, q, l)$ with $k(\geq 1)$ rooted trees $T_{1}, \ldots, T_{k}$ attached. Then, $\operatorname{irr}_{t}(G) \leq(n-p-q-l+2)(n+$ $p+q+l-1)+2 p+2 q+2 l-8$, and the equality holds if and only if $G \cong \infty^{1}\left(p, q, l, S_{r}\right)$ or $G \cong \infty^{5}\left(p, q, l, S_{r}\right)$.

Proof. If $r=1$, then $n=p+q+l-2$, and the rooted trees $T_{1}, \ldots, T_{k}$ are trivial; namely, $\left|V\left(T_{i}\right)\right|=1$ with $i=1, \ldots, k$. Then, $G=\infty(p, q, l)=\infty^{1}\left(p, q, l, S_{1}\right)$ and $\operatorname{irr}_{t}(G)=(n-p-$ $q-l+2)(n+p+q+l-1)+2 p+2 q+2 l-8$.

If $r \geq 2$, we complete the proof by the following three cases.

Case $1\left(G \neq \infty(p, q, l, T)\right.$ where $\left.T \in \mathbb{S}^{*} \cup \mathbb{P} \mathbb{S}^{*}\right)$. Let $G_{1}$ be the graph obtained from $G$ by repeating $\alpha$-transformation, and we cannot get a new graph from $G_{1}$ by $\alpha$-transformation. Then there exists a rooted tree $T$ such that $G_{1} \cong \infty(p, q, l, T)$, where $T \in \mathbb{S}^{*} \bigcup \mathbb{P} \mathbb{S}^{*}$ and $\operatorname{irr}_{t}(G)<\operatorname{irr}_{t}\left(G_{1}\right)$ by Lemma 4 . Let $u$ be the root of the rooted tree $T$.

Subcase $1.1\left(G_{1} \cong \infty(p, q, l, T)\right.$ where $\left.T \in \mathbb{S}^{*}\right)$. If $u=w_{1}$, then $G_{1} \cong \infty^{1}\left(p, q, l, S_{r}\right)$ and $\operatorname{irr}_{t}(G)<\operatorname{irr}_{t}\left(G_{1}\right)=(n-p-q-l+$ 2) $(n+p+q+l-1)+2 p+2 q+2 l-8$.

If $u=w_{5}$, then $G_{1} \cong \infty^{5}\left(p, q, l, S_{r}\right)$ and $\operatorname{irr}_{t}(G)<$ $\operatorname{irr}_{t}\left(G_{1}\right)=(n-p-q-l+2)(n+p+q+l-1)+2 p+2 \mathrm{q}+2 l-8$.

If $u \neq w_{1}$ and $u \neq w_{5}$, then we can get a new graph $G_{2} \cong$ $\infty^{1}\left(p, q, l, S_{r}\right)$ by $\beta$-transformation on $G_{1}$; thus $\operatorname{irr}_{t}(G)<$ $\operatorname{irr}_{t}\left(G_{1}\right)<\operatorname{irr}_{t}\left(G_{2}\right)=(n-p-q-l+2)(n+p+q+l-$ 1) $+2 p+2 q+2 l-8$ by Lemma 6 .

Subcase $1.2\left(G_{1} \cong \infty(p, q, l, T)\right.$ where $\left.T \in \mathbb{P} \mathbb{S}^{*}\right)$. If $u \neq w_{1}$, let $G_{2}$ be the graph obtained from $G_{1}$ by $\beta$-transformation. Then, $\operatorname{irr}_{t}\left(G_{1}\right)<\operatorname{irr}_{t}\left(G_{2}\right)$ by Lemma 5. Repeating $\alpha$-transformation on $G_{2}$ until we cannot get a new graph, the resulting graph is $G_{3} \cong \infty^{1}\left(p, q, l, S_{r}\right)$. By Lemma $4, \operatorname{irr}_{t}\left(G_{2}\right)<\operatorname{irr} r_{t}\left(G_{3}\right)$ and

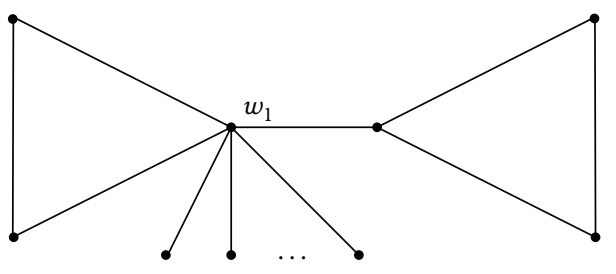

Figure 10: The graph $\infty^{1}\left(3,3,2, S_{n-5}\right)$.

thus $\operatorname{irr}_{t}(G)<\operatorname{irr}_{t}\left(G_{1}\right)<\operatorname{irr}_{t}\left(G_{2}\right)<\operatorname{irr}_{t}\left(G_{3}\right)=(n-p-q-l+$ 2) $(n+p+q+l-1)+2 p+2 q+2 l-8$.

If $u=w_{1}$ and $d_{G_{1}}(u, v)=1$, let $G_{2}$ be the graph obtained from $G_{1}$ by $\beta$-transformation; then $G_{2} \cong \infty^{1}\left(p, q, l, S_{r}\right)$, and $\operatorname{irr}_{t}(G)<\operatorname{irr}_{t}\left(G_{1}\right)<\operatorname{irr}_{t}\left(G_{2}\right)=(n-p-q-l+2)(n+p+q+$ $l-1)+2 p+2 q+2 l-8$ by Lemma 5 .

If $u=w_{1}$ and $d_{G_{1}}(u, v)>1$, let $G_{2}$ be the graph obtained from $G_{1}$ by $\beta$-transformation and let $G_{3}$ be the graph obtained from $G_{2}$ by repeating $\alpha$-transformation until we cannot get a new graph from $G_{3}$ by $\alpha$-transformation. Then by Lemmas 4 and 5, we know that $G_{3} \cong \infty^{1}\left(p, q, l, S_{r}\right)$ and $\operatorname{irr}_{t}(G)<$ $\operatorname{irr}_{t}\left(G_{1}\right)<\operatorname{irr}_{t}\left(G_{2}\right)<\operatorname{irr}_{t}\left(G_{3}\right)=(n-p-q-l+2)(n+p+q+$ $l-1)+2 p+2 q+2 l-8$.

Case $2\left(G \cong \infty(p, q, l, T)\right.$ where $\left.T \in \mathbb{S}^{*}\right)$. The proof is similar to the proof of Subcase 1.1; thus, we omit it.

Case $3\left(G \cong \infty(p, q, l, T)\right.$ where $\left.T \in \mathbb{P S}^{*}\right)$. The proof is similar to the proof of Subcase 1.2; thus, we omit it.

Combining the above arguments, we complete the proof.

Theorem 12. Let $p, q, l, r$ be positive integers with $p, q \geq 3$ and $l \geq 2$. Then,

(1) If $p \geq 4$, then $\operatorname{irr}_{t}\left(\infty^{1}\left(p, q, 1, S_{r}\right)\right)<\operatorname{irr}_{t}\left(\infty^{1}(p-\right.$ $\left.\left.1, q, 1, S_{r+1}\right)\right)$.

(2) If $q \geq 4$, then $\operatorname{irr}_{t}\left(\infty^{1}\left(p, q, 1, S_{r}\right)\right)<\operatorname{irr}_{t}\left(\infty^{1}(p, q-\right.$ $\left.\left.1,1, S_{r+1}\right)\right)$.

(3) If $l \geq 3$, then $\operatorname{irr}_{t}\left(\infty^{1}\left(p, q, l, S_{r}\right)\right)<i r r_{t}\left(\infty^{1}(p, q, l-\right.$ $\left.1, S_{r+1}\right)$ ).

Proof. Clearly, the proofs of (1), (2), and (3) are similar; we only show that (1) holds. Let $n=p+q+l+r-3$; then by formula (14), we have

$$
\begin{aligned}
\operatorname{irr}_{t} & \left(\infty^{1}\left(p-1, q, 1, S_{r+1}\right)\right)-\operatorname{irr}_{t}\left(\infty^{1}\left(p, q, 1, S_{r}\right)\right) \\
\quad & =2 p+2 q+2 l-6>0 .
\end{aligned}
$$

Theorem 13. Let $n \geq 6$ be a positive integer and let $G \in B_{n}^{++}$ be a bicyclic graph on $n$ vertices. Then, $\operatorname{irr}_{t}(G) \leq n^{2}+n-34$ and the equality holds if and only if $G \cong \infty^{1}\left(3,3,2, S_{n-5}\right)$ (see Figure 10).

Proof. Let $n, p, q, l, r$ be positive integers with $p, q \geq 3, l \geq 2$, and $p+q+r+l-3=n$ and let $G \in B_{n}^{++}$be a bicyclic 
graph on $n$ vertices obtained from $\infty(p, q, l)$ with $k(\geq 1)$ rooted trees $T_{1}, \ldots, T_{k}$ attached. Then by Theorem 11, we have $\operatorname{irr}_{t}(G) \leq(n-p-q-l+2)(n+p+q+l-1)+2 p+2 q+2 l-8$, and the equality holds if and only if $G \cong \infty^{1}\left(p, q, l, S_{r}\right)$ or $G \cong \infty^{5}\left(p, q, l, S_{r}\right)$. Note that the results of Theorem 12 also hold for $\infty^{5}\left(p, q, l, S_{r}\right)$; then we only consider the case $\infty^{1}\left(p, q, l, S_{r}\right)$. Now we complete the proof by the following three cases.

Case $1(p=q=3$ and $l=2)$. Then, $\operatorname{irr}_{t}\left(\infty^{1}\left(3,3,2, S_{n-5}\right)\right)=$ $n^{2}+n-34$.

Case 2 ( $p \geq q \geq 3, l \geq 2$, and $p \geq 4)$. Then by (1), (2), and (3) of Theorem 12, we have $\operatorname{irr}_{t}\left(\infty^{1}\left(p, q, l, S_{r}\right)\right)<\operatorname{irr}_{t}\left(\infty^{1}(3, q\right.$, $\left.\left.l, S_{n-q-l}\right)\right) \leq \operatorname{irr}_{t}\left(\infty^{1}\left(3,3, l, S_{n-3-l}\right)\right) \leq \operatorname{irr}_{t}\left(\infty^{1}\left(3,3,2, S_{n-5}\right)\right)$.

Case 3 ( $q \geq p \geq 3, l \geq 2$, and $q \geq 4$ ). The proof is similar to Case 2; thus, we omit it.

3.3. The Graph with the Maximal Total Irregularity in $\Theta_{n}$. In this subsection, the bicyclic graph with the maximal total irregularity in $\Theta_{n}$ is determined.

Let $n, p, q, l, r$ be positive integers with $l \geq 2, p, q \geq l+1$, and $p+q+r-l-1=n ; G=\theta^{1}\left(p, q, l, S_{r}\right)$. Clearly, the degree sequence of $G$ is $(r+2,3,2, \ldots, 2,1, \ldots, 1)$. By simple calculation,

$$
\begin{aligned}
\operatorname{irr}_{t}(G)= & (n-p-q+l)(n+p+q-l+1) \\
& +2 p+2 q-2 l-4 .
\end{aligned}
$$

Theorem 14. Let $n, p, q, l, r$ be positive integers with $l \geq 2$, $p, q \geq l+1$, and $p+q+r-l-1=n$, and let $G \in \Theta_{n}$ be a bicyclic graph on $n$ vertices obtained from $\theta(p, q, l)$ with $k(\geq 1)$ rooted trees $T_{1}, \ldots, T_{k}$ attached. Then, $\operatorname{irr}_{t}(G) \leq(n-$ $p-q+l)(n+p+q-l+1)+2 p+2 q-2 l-4$, and the equality holds if and only if $G \cong \theta^{1}\left(p, q, l, S_{r}\right)$.

Proof. If $r=1$, then $n=p+q-l$, and the rooted trees $T_{1}, \ldots, T_{k}$ are trivial; namely, $\left|V\left(T_{i}\right)\right|=1$ with $i=1, \ldots, k$. Then, $G=\theta(p, q, l)=\theta^{1}\left(p, q, l, S_{1}\right)$ and $\operatorname{irr}_{t}(G)=(n-p-q+$ $l)(n+p+q-l+1)+2 p+2 q-2 l-4$.

If $r \geq 2$, we complete the proof by the following three cases.

Case $1\left(G \not \theta(p, q, l, T)\right.$ where $\left.T \in \mathbb{S}^{*} \cup \mathbb{P} \mathbb{S}^{*}\right)$. Let $G_{1}$ be the graph obtained from $G$ by repeating $\alpha$-transformation, and we cannot get a new graph from $G_{1}$ by $\alpha$-transformation. Then there exists a rooted tree $T$ such that $G_{1} \cong \theta(p, q, l, T)$, where $T \in \mathbb{S}^{*} \cup \mathbb{P} \mathbb{S}^{*}$ and $\operatorname{irr}_{t}(G)<\operatorname{irr}_{t}\left(G_{1}\right)$ by Lemma 4 . Let $u$ be the root of the rooted tree $T$.

Subcase $1.1\left(G_{1} \cong \theta(p, q, l, T)\right.$ where $\left.T \in \mathbb{S}^{*}\right)$. If $u=w_{1}$ or $u=w_{5}$, then $G_{1} \cong \theta^{1}\left(p, q, l, S_{r}\right)$ and $\operatorname{irr}_{t}(G)<\operatorname{irr}_{t}\left(G_{1}\right)=$ $(n-p-q+l)(n+p+q-l+1)+2 p+2 q-2 l-4$.

If $u \neq w_{1}$ and $u \neq w_{5}$, then we can get a new graph $G_{2} \cong$ $\theta^{1}\left(p, q, l, S_{r}\right)$ by $\beta$-transformation on $G_{1}$; thus $\operatorname{irr}_{t}(G)<$
$\operatorname{irr}_{t}\left(G_{1}\right)<\operatorname{irr}_{t}\left(G_{2}\right)=(n-p-q+l)(n+p+q-l+1)+2 p+2 q-2 l-4$ by Lemma 6.

Subcase $1.2\left(G_{1} \cong \theta(p, q, l, T)\right.$ where $\left.T \in \mathbb{P S}^{*}\right)$. If $u \neq w_{1}$, let $G_{2}$ be the graph obtained from $G_{1}$ by $\beta$-transformation. Then, $\operatorname{irr}_{t}\left(G_{1}\right)<\operatorname{irr}_{t}\left(G_{2}\right)$ by Lemma 5. Repeating $\alpha$-transformation on $G_{2}$ until we cannot get a new graph, the resulting graph is $G_{3} \cong \theta^{1}\left(p, q, l, S_{r}\right)$. By Lemma $4, \operatorname{irr}_{t}\left(G_{2}\right)<\operatorname{irr}_{t}\left(G_{3}\right)$ and thus $\operatorname{irr}_{t}(G)<\operatorname{irr}_{t}\left(G_{1}\right)<\operatorname{irr}_{t}\left(G_{2}\right)<\operatorname{irr}_{t}\left(G_{3}\right)=(n-p-q+l)(n+$ $p+q-l+1)+2 p+2 q-2 l-4$.

If $u=w_{1}$ and $d_{G_{1}}(u, v)=1$, let $G_{2}$ be the graph obtained from $G_{1}$ by $\beta$-transformation; then $G_{2} \cong \theta^{1}\left(p, q, l, S_{r}\right)$ and $\operatorname{irr}_{t}(G)<\operatorname{irr}_{t}\left(G_{1}\right)<\operatorname{irr}_{t}\left(G_{2}\right)=(n-p-q+l)(n+p+q-l+$ 1) $+2 p+2 q-2 l-4$ by Lemma 5 .

If $u=w_{1}$ and $d_{G_{1}}(u, v)>1$, let $G_{2}$ be the graph obtained from $G_{1}$ by $\beta$-transformation and let $G_{3}$ be the graph obtained from $G_{2}$ by repeating $\alpha$-transformation until we cannot get a new graph from $G_{3}$ by $\alpha$-transformation. Then by Lemmas 4 and 5, we know that $G_{3} \cong \theta^{1}\left(p, q, l, S_{r}\right)$ and $\operatorname{irr}_{t}(G)<$ $\operatorname{irr}_{t}\left(G_{1}\right)<\operatorname{irr}_{t}\left(G_{2}\right)<\operatorname{irr}_{t}\left(G_{3}\right)=(n-p-q+l)(n+p+$ $q-l+1)+2 p+2 q-2 l-4$.

Case $2\left(G \cong \theta(p, q, l, T)\right.$ where $\left.T \in \mathbb{S}^{*}\right)$. The proof is similar to the proof of Subcase 1.1; thus, we omit it.

Case $3\left(G \cong \theta(p, q, l, T)\right.$ where $\left.T \in \mathbb{P S}^{*}\right)$. The proof is similar to the proof of Subcase 1.2; thus, we omit it.

Combining the above arguments, we complete the proof.

Theorem 15. Let $p, q, l, r$ be positive integers with $l \geq 2$ and $p, q \geq l+1$. Then,

(1) If $p \geq 4$, then $\operatorname{irr}_{t}\left(\theta^{1}\left(p, q, 1, S_{r}\right)\right)<\operatorname{irr}_{t}\left(\theta^{1}(p-1\right.$, $\left.\left.q, 1, S_{r+1}\right)\right)$.

(2) If $q \geq 4$, then $\operatorname{irr}_{t}\left(\theta^{1}\left(p, q, 1, S_{r}\right)\right)<\operatorname{irr}_{t}\left(\theta^{1}(p, q-1\right.$, $\left.\left.1, S_{r+1}\right)\right)$.

(3) If $l \geq 3$, then $\operatorname{irr}_{t}\left(\theta^{1}\left(p, q, l, S_{r}\right)\right)<\operatorname{irr}_{t}\left(\theta^{1}(p-1, q-\right.$ $\left.\left.1, l-1, S_{r+1}\right)\right)$.

Proof. Clearly, the proofs of (1), (2), and (3) are similar; we only show that (1) holds. Let $n=p+q+l+r-3$; then by formula (14), we have

$$
\begin{aligned}
& \operatorname{irr}_{t}\left(\theta^{1}\left(p-1, q, 1, S_{r+1}\right)\right)-\operatorname{irr}_{t}\left(\theta^{1}\left(p, q, 1, S_{r}\right)\right) \\
& \quad=2 p+2 q-2 l-2>0 .
\end{aligned}
$$

Theorem 16. Let $n \geq 4$ be a positive integer and let $G \in \Theta_{n}$ be a bicyclic graph on $n$ vertices. Then, $\operatorname{irr}_{t}(G) \leq n^{2}+n-16$ and the equality holds if and only if $G \cong \theta^{1}\left(3,3,2, S_{n-3}\right)$ (see Figure 11).

Proof. Let $n, p, q, l, r$ be positive integers with $l \geq 2, p, q \geq$ $l+1$, and $p+q+r-l-1=n$ and let $G \in \Theta_{n}$ be a bicyclic graph on $n$ vertices obtained from $\theta(p, q, l)$ with $k(\geq 1)$ rooted trees $T_{1}, \ldots, T_{k}$ attached. Then by Theorem 14 , we have 


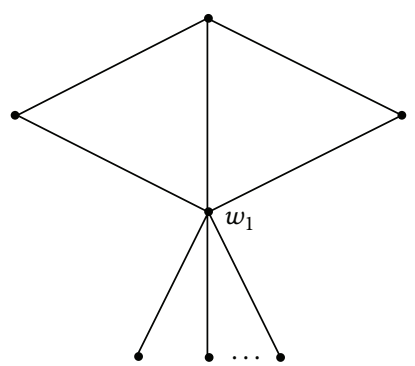

Figure 11: The graph $\theta^{1}\left(3,3,2, S_{n-3}\right)$.

$\operatorname{irr}_{t}(G) \leq(n-p-q+l)(n+p+q-l+1)+2 p+2 q-2 l-4$, and the equality holds if and only if $G \cong \theta^{1}\left(p, q, l, S_{r}\right)$. Now we complete the proof by the following three cases.

Case $1(p=q=3$ and $l=2)$. Then, $\operatorname{irr}_{t}\left(\theta^{1}\left(3,3,2, S_{n-3}\right)\right)=$ $n^{2}+n-16$.

Case $2(p \geq q \geq l+1, l \geq 2$ except for $p=q=l+1=3)$

Subcase $2.1(p>l+1, l=2)$. Then by (1) and (2) of Theorem 15, we have $\operatorname{irr}_{t}\left(\theta^{1}\left(p, q, 2, S_{r}\right)\right)<$ $\operatorname{irr}_{t}\left(\theta^{1}\left(3, q, 2, S_{p+r-3}\right)\right) \leq \operatorname{irr}_{t}\left(\theta^{1}\left(3,3,2, S_{n-3}\right)\right)$.

Subcase $2.2(p \geq l+1, l \geq 3)$. Then by (3), (1), and (2) of Theorem 15, we have $\operatorname{irr}_{t}\left(\theta^{1}\left(p, q, l, S_{r}\right)\right)<\operatorname{irr}_{t}\left(\theta^{1}(p-l+\right.$ $\left.\left.2, q-l+2,2, S_{r+l-2}\right)\right) \leq \operatorname{irr}_{t}\left(\theta^{1}\left(3, q-l+2,2, S_{p+r-3}\right)\right) \leq$ $\operatorname{irr}_{t}\left(\theta^{1}\left(3,3,2, S_{n-3}\right)\right)$.

Case $3(q \geq p \geq l+1, l \geq 2$ except for $p=q=l+1=3)$.

The proof is similar to Case 2; thus, we omit it.

3.4. Bicyclic Graphs with the Maximal Total Irregularity. By $\mathscr{B}_{n}=B_{n}^{+} \cup B_{n}^{++} \cup \Theta_{n}$ and Theorems 10, 13, and 16, the following result is obvious.

Theorem 17. Let $n \geq 4$ be a positive integer and let $G \in \mathscr{B}_{n}$ be a bicyclic graph on $n$ vertices. Then, $\operatorname{irr}_{t}(G) \leq n^{2}+n-16$ and the equality holds if and only if $G \cong \theta^{1}\left(3,3,2, S_{n-3}\right)$ (see Figure 11).

\section{Conflict of Interests}

The authors declare that there is no conflict of interests regarding the publication of this paper.

\section{Acknowledgments}

The authors would like to thank the referees for their valuable comments, corrections, and suggestions, which lead to the improvement of the original paper. This research is supported by National Natural Science Foundation of China (no. 11301093), the Zhujiang Technology New Star Foundation of Guangzhou (no. 2011J2200090), and Program on International Cooperation and Innovation, Department of Education, Guangdong Province (no. 2012gjhz0007).

\section{References}

[1] M. O. Albertson, “The irregularity of a graph," Ars Combinatoria, vol. 46, pp. 219-225, 1997.

[2] H. Abdo, N. Cohen, and D. Dimitrov, "Bounds and computation of irregularity of a graph," Filomat. In press.

[3] M. A. Henning and D. Rautenbach, "On the irregularity of bipartite graphs," Discrete Mathematics, vol. 307, no. 11-12, pp. 1467-1472, 2007.

[4] P. Hansen and H. Mèlot, Variable neighborhood search for extremal graphs. 9. Bounding the irregularity of a graph, vol. 69 of DIMACS Series in Discrete Mathematics and Theoretical Computer Science, 2005.

[5] H. Abdo and D. Dimitrov, "The total irregularity of a graph," http://arxiv.org/abs/1207.5267.

[6] D. Dimitrov and R. Škrekovski, "Comparing the irregularity and the total irregularity of graphs," Ars Mathematica Contemporanea. In press.

[7] L. H. You, J. S. Yang, and Z. F. You, "The maximal total irregularity of unicyclic graphs," Ars Combinatoria. In press.

[8] Y. X. Zhu, L. H. You, and J. S. Yang, "The minimal total irregularity of graphs," http://arxiv.org/abs/1404.0931.

[9] H. Abdo and D. Dimitrov, "The total irregularity of graphs under graph operations," http://arxiv.org/abs/1304.0185.

[10] J. A. Bondy and U. S. R. Murty, Graph Theory with Applications, MacMillan, London, UK, 1976. 


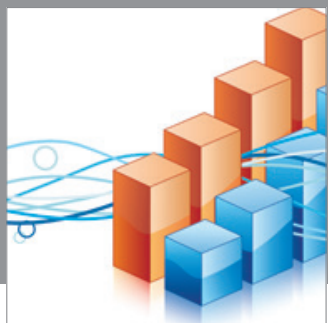

Advances in

Operations Research

mansans

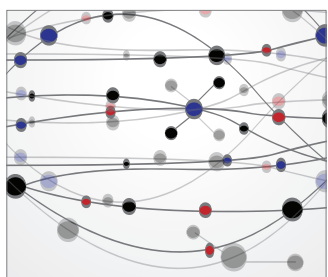

The Scientific World Journal
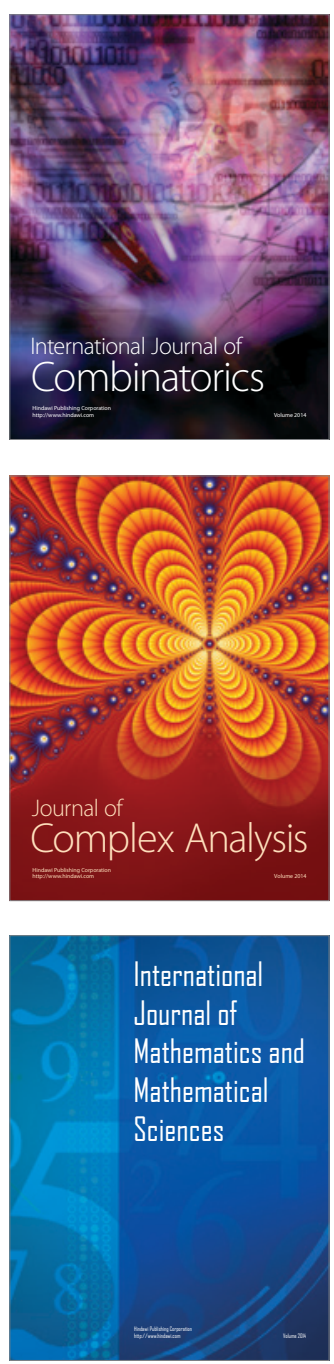
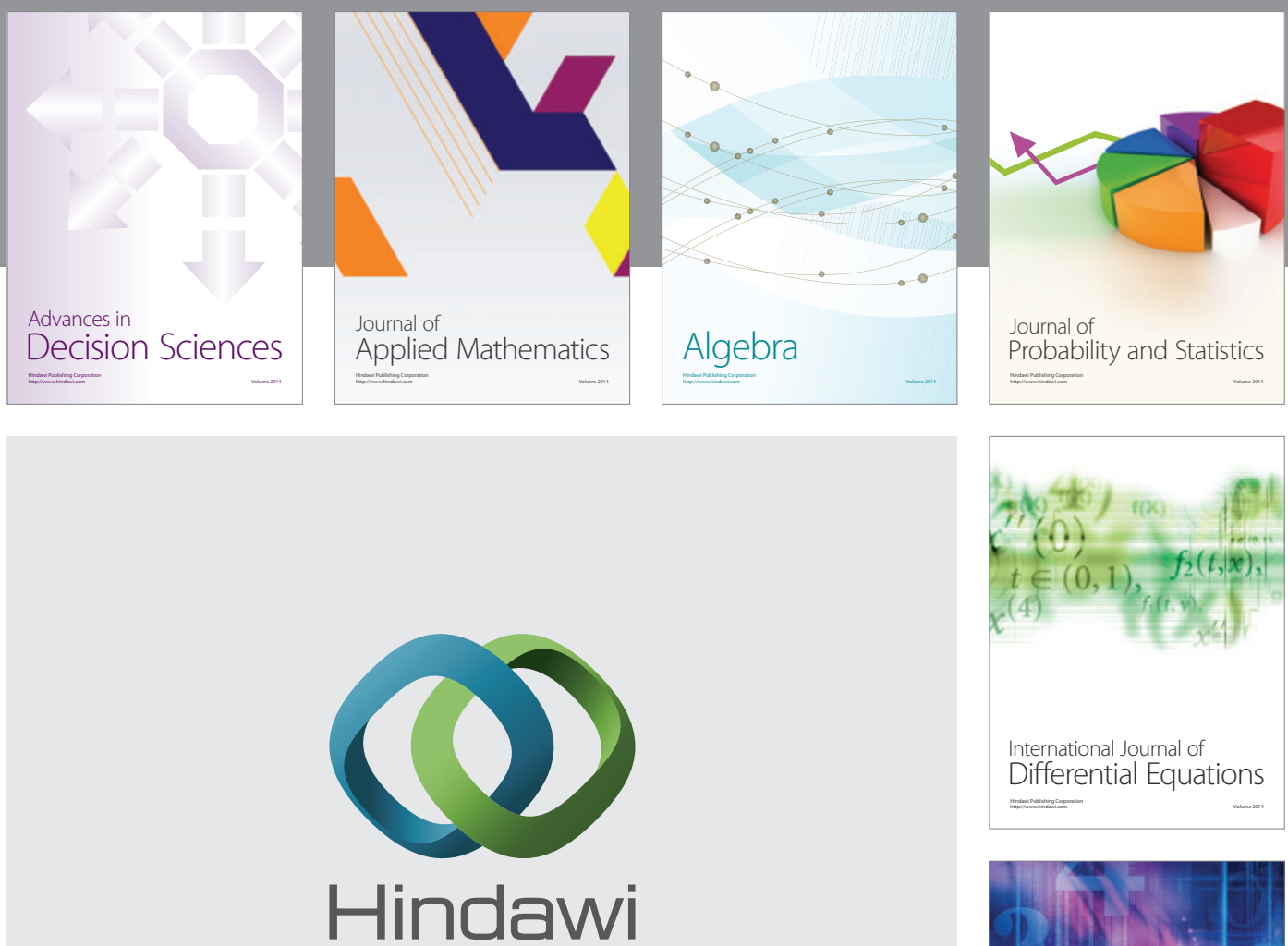

Submit your manuscripts at http://www.hindawi.com
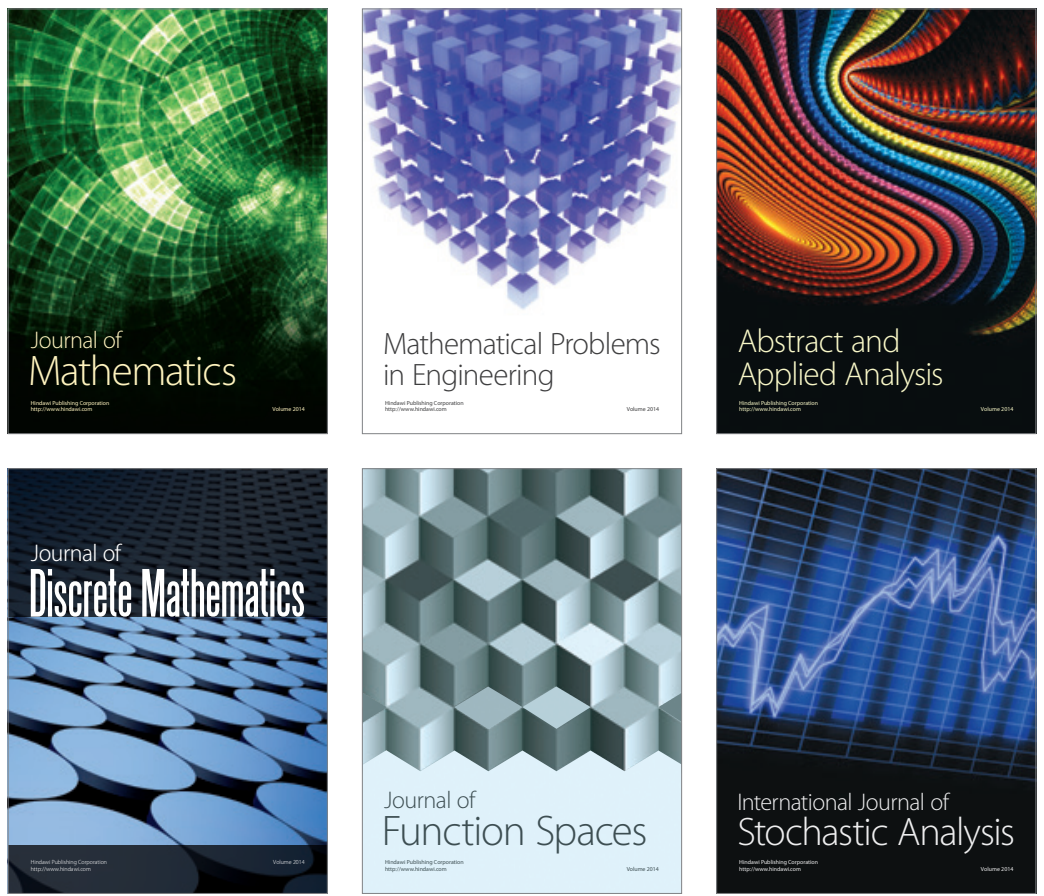

Journal of

Function Spaces

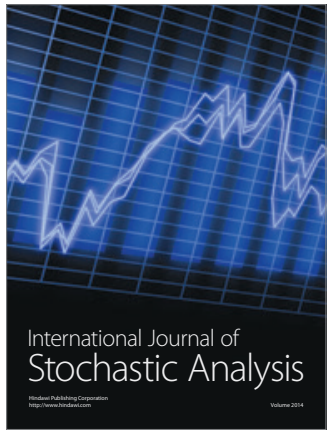

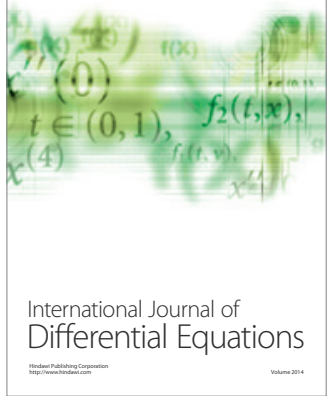
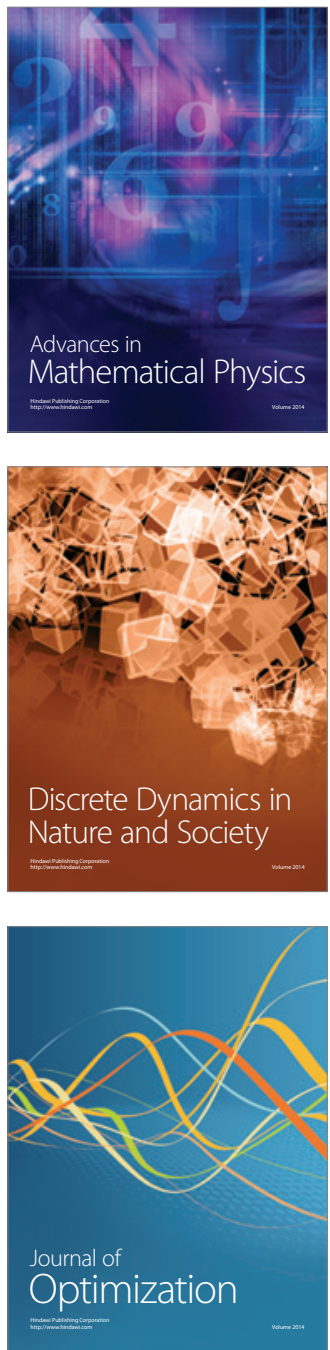